\title{
Addiction, Experimental Models and Neurobiological Mechanisms
}

\author{
Régis Bordet \\ Département de Pharmacologie Médicale, Centre d'Évaluation et d'Information sur la Pharmacoépendance, Faculté de Médecine, Lille, \\ France
}

Text received September $2^{\text {th }}, 2014$; accepted September $30^{\text {th }}, 2014$

\begin{abstract}
Keywords:
addiction;

pharmacology;

neurobiology;

animal models;

social impact

Abstract - Humans have constantly sought to alleviate their existential anxieties, first resorting to substances found in their natural environment, and more recently, with the arrival of modern chemistry, using synthetic substances or medications. The substances used in this way are constantly renewing, warranting health surveillance and particular vigilance towards the addictive risk, given its major medical and social impact. This surveillance and vigilance requires detailed, accurate knowledge of the pharmacological and physio-pathological models involved in the emergence of the process of addiction, in particular disturbances of the systems regulating dopaminergic transfer; it also requires knowledge of the means to identify individual risk factors linked to genetic or psycho-behavioural susceptibilities.
\end{abstract}

Abbreviations: see end of article.

\section{Introduction}

Since time began, humans have sought the means to alleviate their existential anxieties, first using substances found in the natural environment, and more recently, with the arrival of modern chemistry, using synthetic substances or medications. The use of these substances procures pleasurable feelings via the activation of the reward circuit, where the main agent is dopamine. While this usage is at the outset most often recreational, or indeed therapeutic, this purpose is sometimes overtaken, and the subject becomes dependant on the substance involved, experiencing physical and mental pain in case of abstinence, craving, and an irrepressible desire to resume its use. The ephemeral pleasure derived from substance use is gradually replaced by the sole need to have it, with social and medical consequences that mean suffering for the subject and those around him or her. ${ }^{[1,3]}$ The substances that are used or misused are constantly renewing, which requires health surveillance measures, in particular towards the risk of addiction. This surveillance and monitoring requires detailed knowledge of the physio-pathological mechanisms, of models of the dependency process, and of the means to detect addiction risk.

\section{Addiction: from natural substances to synthetic psychotropics}

Humans soon found the means in their natural environment to alter their brain functioning and induce affective, emotional and cognitive effects generating feelings of pleasure, thus relieving or obliterating existential human suffering. There are numerous examples of this quest: cannabis, bethel nuts, hallucinogenic mushrooms, coca leaves, cape gooseberries, poppy flowers and others. ${ }^{[4,5]}$ When nature alone no longer sufficed, people began to modify these substances to obtain substances with psychotropic properties. The best example of this is alcohol, the first instance of the impact of a chemical alteration, here by way of fermentation. It was however only after the development of extraction processes in the $18^{\text {th }}$ century that the active substances responsible for psychotropic effects were progressively identified. The identification of the relevant molecules made the mechanisms involved easier to apprehend, so that there was a shift from a rather mystic view of these substances, as in Baudelaire's phrase "artificial paradises", to a more scientific view which opened the way to neuropsycho-pharmacology. ${ }^{[6]}$ Naturally, the improving knowledge of the mechanisms of action of the "natural" psychotropics opened 
the way to synthetic psychoactive drugs, where the purpose was initially therapeutic, but the usage of these substances was then diverted towards illicit usage, or uses for which the drugs were not initially intended.

Different preparations based on morphine were used, for pain control purposes or to sedate agitated patients, in oral form and later in injectable form. The search for new cough medicines based on morphine, rapidly known for its respiratory depression effects, led to the synthesis of diacetylmorphine, referred to as a "heroic" drug by its developer. Thus heroin was born, marketed in 1898 as a cough medicine, but its strong addictive properties soon led to usage that was primarily illicit rather than therapeutic. ${ }^{[7}$ Amphetamines are linked to the development of bronchodilators such as ephedrine, the psycho-stimulant properties of which were very soon recognised. This led to numerous other amphetamine-based substances, the most recent of which is methylenedioxymethamphetamine (MDMA) or ecstasy. ${ }^{[8,9]}$ The history of tranquillizers is closely linked to their muscle-relaxant effect. It was indeed when looking for antibiotics that Franck Berger came upon mephenesin because he noticed its muscle-relaxant effect on laboratory mice. ${ }^{[10]}$ A substance derived from mephenesin, meprobamate $\left(\right.$ Equanil $\left.{ }^{\circledR}\right)$ was the first tranquillizer marketed in 1955 , but it was rapidly rivalled by the benzodiazepines, the first of which, chlordiazepoxide $\left(\right.$ Librium ${ }^{\circledR}$ ) was developed in 1958 by Leo Sternbach. ${ }^{[11]}$ It was from the structure of a phenothiazine, which produced chlorpromazine, the first antipsychotic, and imipramine, the first antidepressant, that chlordiazepoxide was developed. Its metabolite, diazepam $\left(\right.$ Valium $^{\circledR}$ ) was for some years the world leader in tranquillizers following its marketing in 1963. The anxiolytic effect rapidly took precedence over it mere muscle-relaxant effect, which explains the popularity of this class of psychotropics and its over-prescription. Other substances, such as lysergic acid diethylamide (LSD) or phencyclidine, were substances that were specifically aimed at "recreational" use, and were the not subject to pharmaco-therapeutic development.

If we consider the effects of these different substances, it becomes obvious that the boundary between recreational usage and risk of addiction is not clear-cut. Indeed, it is the recreational aspect, the sensation of pleasure or the reduction of anxiety, rather than dependency, that are sought after by users. Nevertheless the neurobiological effect on the reward system, often not the main mechanisms of action, coupled with repeated usage are what lead to deregulation of the motivation and reward circuits, inhibiting control, and memorisation which underpin dependency and addiction. ${ }^{[12]}$ Different models, whether pharmacological, experimental or neurobiological, give insight into the shift from recreational use to loss of control and compulsive use, the main characteristics of dependency and addiction. Addiction also needs to be envisaged at individual level via a model of interaction between genetic susceptibility and psycho-behavioural vulnerability, and at collective level via the modelling of its social impact.

\section{Addiction: the pharmacological model}

Progress in the field of neuro-psycho-pharmacology and in molecular biology has in the space of a few decades made it possible to characterise the cellular and molecular targets of psychotropic substances, whether therapeutic or illicit. ${ }^{[13-15]}$ The receptor targets and their regional or cellular locations are numerous, and explain the diversity of the pharmacodynamic effects produced. Certain substances act through neurone activator systems, thus explaining i) psychostimulant effects (psycho-motor excitation) in particular via the activation of the mono-aminergic systems by increasing their release (amphetamines, methylphenidate, ecstasy), or by inhibition of their reuptake (cocaine, antidepressants), or via the cholinergic system and its nicotine receptors; ii) psychodysleptic effects, through interaction with certain subtypes of glutamate receptors (phencyclidine), with cannabinoid receptors (cannabis), and with certain subtypes of serotoninergic receptors (LSD, mescaline). In contrast, other substances act on systems that are inhibitors of neurone activity, explaining their anxiolytic effect and the corollary de-inhibition effect. This is the case with alcohol, barbiturates, or benzodiazepines, which act via a receptor complex that is sensitive to gamma-aminobutyric acid (GABA). The modulation of opioid receptors by opiates (morphine and derived substances, heroin) produces both positive effects such as feelings of well-being and euphoria, sometimes bordering on something resembling an orgasm, and pain-inhibiting effects which also contribute to the hedonic effect.

While the main mechanisms of all these addictive substances are diverse, the existence of hedonic properties that are fairly similar and lead to repeated use in order to retrieve the sensations suggests a possible shared final pathway. Experiments in primates and rodents have enabled the identification of reward circuits which, when stimulated, produce pleasure, driving the animal to seek to return to the circumstances in which this brain system is electrically activated. ${ }^{[12,16]}$ This ability to reinforce the activation of the reward system is also procured by natural or synthetic substances, which explains repeated use of the substance to retrieve the pleasure reinforcement effect. In this paradigm, the notion of pleasure includes both the emotional aspect (the hedonic sensation) and the motivational aspect (the activity of sensationseeking) [figure 1]. From an anatomo-functional viewpoint, this reward circuit is located in the ventral tegmental area, which is a small zone situated in the upper brainstem close to the substantia nigra controlling movement. Its neurones project towards the nucleus accumbens, a striatum zone linked to the limbic system and 


\section{Mesolimbic pathway $=$ Reward and pleasure circuit}

\section{Psychoactive substance}

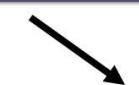

Mesolimbique pathway

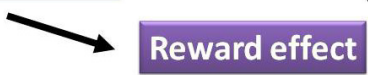

Fig. 1. The reward circuit and reinforcement potential.

hence to emotional functions, and towards the prefrontal cortex which is involved in motivational processes. ${ }^{[17]}$

From a neurobiological viewpoint, dopamine is the main agent since it is synthesised in the neurones of the ventral tegmental area and released into the nucleus accumbens and the prefrontal cortex. A relationship has been clearly established between the activation of this meso-corticolimbic system and the release of dopamine in its target zones. ${ }^{[18,19]}$ With the progress in imagery, in microdialysis experiments in animals and more recently in humans it has also been shown that all the substances described as "artificial paradises" are able to activate this pathway and trigger the release of dopamine. For instance, a link has been clearly established between the inducement of a euphoric effect by amphetamines and the release of dopamine it produces. The activation of a final common pathway might appear surprising or even paradoxical given the diversity of the main mechanisms of action of psychoactive substances. While it is easy to apprehend for substances such as amphetamines or cocaine, which act directly on the dopaminergic system, it is less obvious for substances that act on other neurotransmitter systems. However cellular and molecular biology has made it possible to evidence the fact that all the receptor targets are expressed in the cellular structure of the dopaminergic neurones in the ventral tegmental area, where they are capable of regulating the release of dopamine in the nucleus accumbens.

\section{Addiction: the experimental models}

The experimental models of addiction are based on the observation of effects in animals of repeated exposure to addictive substances. ${ }^{[20,21]}$ More recently, genetic models have appeared, underpinned by the identification of genes linked to susceptibility towards addiction. ${ }^{[22]}$ However no single model enables all the physio-pathological dimensions of addiction to be represented. The animal models used are rodents, non-human primates, and more recently invertebrates (drosophila, zebra fish, nematodes). ${ }^{[23]}$ This experimental modelling provides important data for determining addictive potential, and should be integrated into expertise in abuse monitoring programmes.

The main line of approach in the modelling of an addiction is based on ad lib administration by the animal, so as to reflect the voluntary nature of substance use in humans, in turn a reflection of the positive learning reinforcement it brings about. These models are sometimes difficult to implement, and require particular experimental devices, as in the case of alcohol which requires administration in vapour form. ${ }^{[24,25]}$ These self-administration models also raise the problem of the absence of choice for the animal, although once it has been receiving the substance over a period of time, the propensity on the part of the animal to choose that substance rather than another neutral substance is greater, so that a Pavlov-type mechanism and a form of conditioning may be involved. These tests based on self-administration can be improved by including indicators (sound or light signals) associated with intake of the substance, where the mere perception by the animal of the signal leads it to seek out the substance, causing positive learning reinforcement. The same applies to stress environments (electric shock, food deprivation) which drive the animal to consume so as to alleviate the negative effects. These considerations have led to more sophisticated models using place preference paradigms, whereby the animal receives the addictive substance and the neutral substance in distinct environments (compartments of different colours for example), and is then faced with an environment in which it has a choice. ${ }^{[26]}$ Models can also combine place preference and indices.

The phenomenon of behavioural sensitisation makes it possible to model motivational processes involved in the quest for a psychoactive substance, which can become established in the course of the addiction process. ${ }^{[26]}$ It mainly concerns locomotor behaviour, although certain forms of stereotypy can also be sensitive to the effect. It shows up in a gradual increase in the intensity of the behavioural response as doses equivalent to the first are repeated. The cellular and molecular exploration of this model has provided a better picture of the neurobiological adaptation mechanisms and the plasticity of the reward system, which lead to deregulation and addiction. This behavioural sensitisation model can constitute an easily implemented test to identify the addictive potential of a substance not hitherto characterised as possessing any.

The administration of a substance enables not only its addictive potential to be known, but also provides useful information on the manifestations that follow on from its withdrawal, since the animal may have physical and behavioural (anxious) 


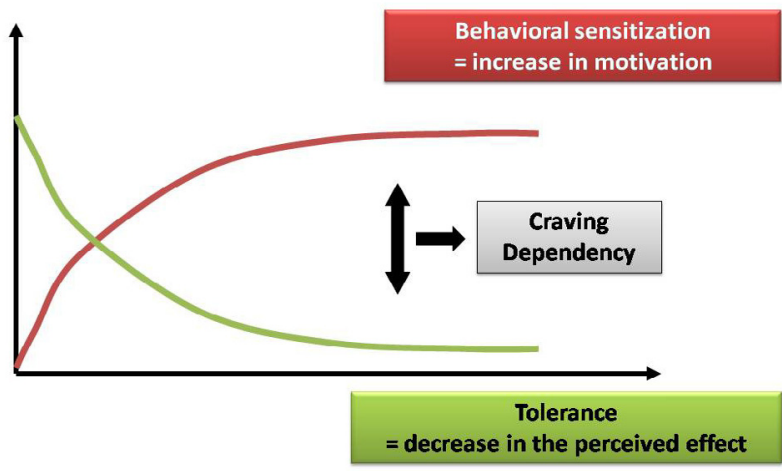

Fig. 2. The psycho-behavioural mechanisms of addiction.

manifestations reflecting its state of dependency. It is possible to model relapse after a period of abstinence, triggering it by readministration of a dose, by stress, or by perception of a signal which will be sufficient to reinstate seeking or self-administration behaviours. ${ }^{[27]}$

\section{Addiction: the neurobiological deregulation model}

The neurobiological reward system exists in biological terms, since it is stimulated by natural actions, such as feeding or the sexual act, relating to the finality of the survival of the species. It ensures that feeding, which enables individual survival, and sexual activity, which enables the survival of the species, are experienced pleasurably, and not merely repetitively, which could lead to interest declining, and a form of anorexia or abstinence that would be harmful for the survival of the species. This system cannot however cope with artificial stimulation, in particular by way of chemical substances, where the level of activation is well above what can be induced physiologically. Excessive, repeated activation of the reward system leads to its deregulation, which takes the form of addiction, that is to say the loss of freedom of choice in usage, the most characteristic phenomenon being the craving effect. ${ }^{[12,26]}$

Different mechanisms enter into the craving phenomenon (figure 2). The first is underpinned by memory processes, whereby the individual remembers the effects experienced from use of the substance, and above all the setting in which usage and the appearance of the effects occurs. Memorisation explains why merely seeing contextual elements of substance use can trigger craving in a dependent subject, resulting in relapse in a subject who may have been abstinent for several years. Brain imagery experiments have enabled the alterations in the reward system to be visualised following viewing of contextual elements of substance use. ${ }^{[28,29]}$ The second mechanism concerns motivational processes, which undergo a sensitisation phenomenon. This

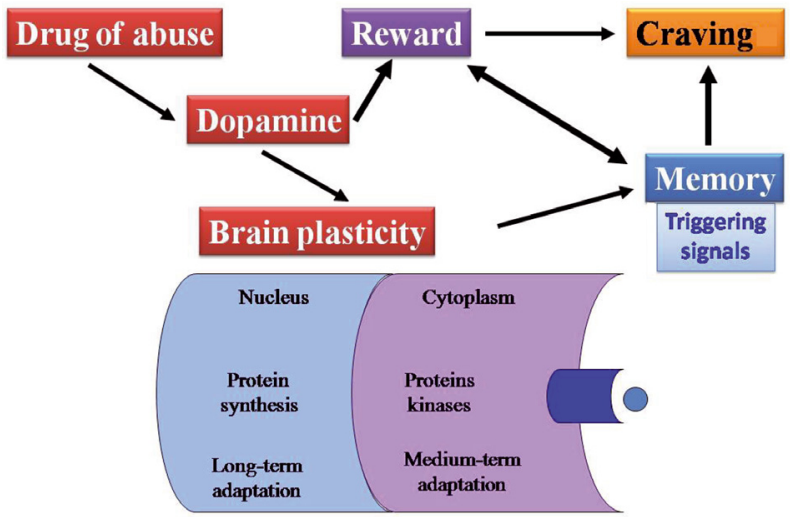

Fig. 3. The neurobiological mechanisms of addiction.

behavioural sensitisation is evidenced experimentally by a gradual increase in the animal's locomotor activity with the repeated administrations, without any need to increase the dose. This sensitisation is accompanied by a behaviour that is centred on obtaining the substance, at the expense of all other activities. ${ }^{[26]}$ The third mechanism concerns tolerance, that is to say the gradual decrease of the pharmacodynamic effect as usage is repeated, resulting in the need to increase the dose to reproduce effects of the same intensity. This tolerance process is linked above all to the reinforcing potential, that is to say the pleasure derived from use of the substance. ${ }^{[19]}$ These three mechanisms underpin addiction and the craving phenomenon, since signals relating to the setting of the substance use trigger the motivation to resume use because of the hypersensitivity of the system, in spite of the fact that the experience is of lesser intensity because of the tolerance phenomenon.

The loss of inhibiting control, which is related to the prefrontal cortex, is an important element in the impulsive and compulsive nature of substance use, and it contributes to dissociation between the motivational dimension, which strengthens, and the hedonic dimension, which declines. The subject is no longer capable of disengaging from the conditioned reflex that is memorised as a result of the initial association between use of the substance and positive reinforcement learning, despite the gradual loss of this reinforcement potential. ${ }^{[30,31]}$ This inability to escape from the conditioned reflex reflects an absence or loss of the cognitive flexibility required to control impulsiveness. This loss of control involves different areas of the brain (orbito-frontal cortex, striatum, medial temporal lobe) and different neurotransmitters (serotonin, dopamine, noradrenaline) the functioning of which changes as a result of repeated substance use. Neuro-imagery and brain stimulation data have shown that a decrease in activity in the orbito-frontal cortex in addiction can at least partially explain the loss of control with regard to substance use.

Neurobiological mechanisms underpin these psychobehavioural mechanisms (figure 3). They result in particular 
from processes of cellular and molecular plasticity linked to the pulsatile nature of the stimulation of the reward circuit. ${ }^{[17]}$ This is particularly true of the dopaminergic pathways, with changes in density and sensitivity of the dopaminergic receptors, which among other things explain the phenomena of behavioural sensitisation and tolerance. They also concern the glutamatergic system, linked to memorisation. But other neurobiological systems appear to play an important part, in particular the serotoninergic and noradrenergic pathways, as do alterations in the opioid system. ${ }^{[32]}$ The association between stress and addiction risk has also led to the exploration of the implication of the corticotropic system, which is indeed modified in addiction models. These molecular targets are all pharmacological targets, despite the fact that aetio-pathogenic treatments aimed specifically at the craving phenomenon are still scarce.

\section{Addiction: a model of individual susceptibility}

The use of potentially addictive substances does not necessarily lead to the development of an addiction. Some individuals may feel aversion on their first encounter with a substance supposed to afford a pleasurable experience. Others may use the substance occasionally without being really dependent, with use being linked to a creative, festive or recreational setting, which fits the definition of an artificial paradise. But for reasons of individual susceptibility, occasional use can shift to repeated use, accompanied by a compulsive quest for the substance and the imperious need to consume, which then signals the addictive pathology. This inter-individual variability can be explained by interaction between genes and the environment.

Genetic susceptibility may indeed be involved, as suggested by family or twin studies, but its influence appears to vary with the substance involved. In the Genome-Wilde Association Studies large cohorts have been used to study genetic variations in the nicotine receptors, serotonin transporters and receptors, circadian patterns, certain ion channels, $\mathrm{P} 450$ cytochrome, and enzymes involved in the catabolism of monoamines. Some of the genetic variations identified are more specific to certain types of addiction, and their presence is associated with alterations in cerebral function. ${ }^{[33]}$ However the physiopathology of addiction cannot be reduced to mere genetic susceptibility, and other exogenous factors (pre- or post-natal stress, unfavourable living conditions etc...) or endogenous factors (impulsivity, cognitive disorders) can also be involved. ${ }^{[34]}$ We are indeed dealing with an interaction between genetic composition and an environment, where environmental factors will reveal or trigger addiction in a genetically predisposing terrain. The use of the substance itself is liable to alter these gene-environment interactions, in an epigenetic perspective on addiction which suggests the possibility of trans-generational transmission, independently from the issue of a recessive autosome. It has also been clearly shown that addictive substances can modulate certain pathways, in particular histone pathways, involved in epigenetic modifications. ${ }^{[35,36]}$

\section{Addiction: a social medicine model}

Addiction is a genuine illness, not a vice, since the substances intended to create an artificial heaven progressively draw individuals who become dependent towards a living hell of physical, mental and social suffering. The first social dimension concerns the subject, who little by little abandons his or her activities and social relationships in favour of an all-consuming quest for the substance. From this point of view, addiction is a model of social withdrawal or even de-socialisation, which explains why medicosocial care is at least as important as medical care. ${ }^{[37,38]}$

The second dimension concerns the way in which society views the addiction. There is social acceptance of certain types of substance use (alcohol or tobacco), indifference towards or even valorisation of some others (cannabis, cocaine), there may be medical supervision (benzodiazepines), while other types of substance use are stigmatised (opiates). Addiction has links with our representations of pleasure or conversely of shamefulness in Western societies, with a continual oscillation between recreational functions and loss of free will. ${ }^{[39]}$ The individual is not the only person involved, and the social impact is very real given the consequences in terms of morbidity and mortality, and also in terms of family life, the economy and the medical and legal systems. Addiction also has a political dimension that is likewise ambivalent - between endeavouring to reduce the phenomenon and reaping the secondary benefits of reducing generalised social anxiety. Addiction poses major challenges for prevention, the organisation of care and research, despite the undeniable progress already achieved. ${ }^{[40]}$ The early identification by the addiction vigilance system of potential risks of abuse of different substances, whether or not they are known for their addictive potential, is part of social care provision, using all the different models available for the understanding of addiction.

Conflicts of interest. None.

Abbreviations. GABA: gamma-aminobutyric acid; LSD: lysergic acid diethylamide; MDMA : methylenedioxymethamphetamine.

\section{References}

1. Ross S, Peselow E. The neurobiology of addictive disorders. Clin Neuropharmacol 2009; $32: 269-76$ 
2. George O, Le Moal M, Koob GF. Allostatis and addiction: role of dopamine and corticotrophin-releasing factor systems. Physiol Behav 2012; 106 : 58-64

3. Mameli M, Lüscher C. Synaptic plasticity and addiction: learning mechanisms gone awry. Neuropharmacology 2011; 61: 1052-9

4. Stolberg VB. The use of coca: prehistory, history, and ethnography. J Ethn Subst Abuse 2011; 10: 126-46

5. Nichols DE. Hallucinogens. Pharmacol Ther 2004; 101: 131-81

6. Lingford-Hughes A, Watson B, Kalk N, et al. Neuropharmacology of addiction and how it informs treatment. Br Med Bull 2010; 96: 93-110

7. Way EL. History of opiate use in the Orient and the United States. Ann N Y Acad Sci 1982; 398: 12-23

8. Wood S, Sage JR, Shuman T, et al. Psychostimulants and cognition: a continuum of behavioral and cognitive activation. Pharmacol Rev 2013; 66: 193-221

9. Bernschneider-Reif S, Oxler F, Freudenmann RW. The origin of MDMA ("ecstasy")-separating the facts from the myth. Pharmazie 2006; 61: 966-72

10. Ban TA. The role of serendipity in drug discovery. Dialogues Clin Neurosci 2006; 8: 335-44

11. Lopez-Munoz F, Alamo C, García-García P. The discovery of chlordiazepoxide and the clinical introduction of benzodiazepines: half a century of anxiolytic drugs. J Anxiety Disord 2011; 25: 554-62

12. Berridge $\mathrm{KC}$, Robinson TE, Aldridge JW. Dissecting components of reward: "liking", "wanting", and learning. Curr Opin Pharmacol 2009; 9 : 65-73

13. Aguilar MA, Rodríguez-Arias M, Miñarro J. Neurobiological mechanisms of the reinstatement of drug-conditioned place preference. Brain Res Rev 2009; $59: 253-77$

14. Cui C, Noronha A, Morikawa $\mathrm{H}$, et al. New insights on neurobiological mechanisms underlying addiction. Neuropharmacology 2013; 67: 223-32

15. Bossert JM, Marchant NJ, Calu DJ, et al. The reinstatement model of drug relapse: recent neurobiological findings, emerging research topics, and translational research. Psychopharmacology 2013; 229: 453-76

16. Rømer Thomsen K, Fjordback LO, Moller A, et al. Applying incentive sensitization models to behavioral addiction. Neurosci Biobehav Rev 2014 Sep; 45: 349-9

17. Steketee JD, Kalivas PW. Drug wanting: behavioral sensitization and relapse to drug-seeking behavior. Pharmacol Rev 2011; 63: 348-65

18. Leyton M, Vezina P. Dopamine ups and downs in vulnerability to addictions: a neurodevelopmental model. Trends Pharmacol Sci 2014; 35: 268-76

19. George O, Le Moal M, Koob GF. Allostasis and addiction: role of the dopamine and corticotropin-releasing factor systems. Physiol Behav 2012; 106: 58-64

20. Fernando ABP, Robbins TW. Animal models of neuropsychiatric disorders. Annu Rev Clin Psychol 2011; 7: 39-61

21. Ahmed SH. The science of making drug-addicted animals. Neuroscience 2012; 211: 107-25

22. Fowler CD, Kenny PJ. Utility of genetically modified mice for understanding the neurobiology of substance use disorders. Hum Genet 2012; 131: 941-57
23. Søvik E, Barron AB. Invertebrate models in addiction research. Brain Behav Evol 2013; 82: 153-65

24. Hopf FW, Lesscher HMB. Rodent models for compulsive alcohol intake. Alcohol 2014; 48: 253-64

25. Marchant NJ, Li X, Shaham Y. Recent developments in animal models of drug relapse. Curr Opin Neurobiol 2013; 23: 675-83

26. Skinner MD, Aubin HJ. Craving's place in addiction theory: contributions of the major models. Neurosci Biobehav Rev 2010; 34: 606-23

27. Perry CJ, Zbukvic I, Kim JH, et al. Role of cues and contexts on drug-seeking behavior. Br J Pharmacol 2014 oct; 171(20): 4636-72

28. Izquierdo A, Jentsch JD. Reversal learning as a measure of impulsive and compulsive behavior in addictions. Psychopharmacology 2012; 219: $607-20$

29. Robinson MJF, Berridge KC. Instant transformation of learned repulsion into motivational "wanting". Curr Biol 2013; 23: 282-9

30. Feil J, Sheppard D, Fitzgerald PB, et al. Addiction, compulsive drug seeking, and the role of frontostriatal mechanisms in regulating inhibitory control Neurosci Biobehav Rev 2010; 35: 248-75

31. Linnet J. Neurobiological underpinnings of reward anticipation and outcome evaluation in gambling disorder. Front Behav Neurosci 2014; 8: 100

32. Tassin JP. Uncoupling between noradrenergic and serotonergic neurons as a molecular basis of stable changes in behavior induced by repeated drugs of abuse. Biochem Pharmacol 2008; 75: 85-97

33. Ducci F, Goldman D. The genetic basis of addictive disorders. Psychiatr Clin North Am 2012; 35: 495-519

34. Enoch MA. The influence of gene-environment interactions on the development of alcoholism and drug dependence. Curr Psychiatry Rep 2012; 14(2) $150-8$

35. Vassoler FM, Byrnes EM, Pierce RC. The impact of exposure to addictive drugs on future generations: physiological and behavioral effects Neuropharmacology 2014; 76: 269-75

36. Vassoler FM, Sadri-Vakili G. Mechanisms of transgenerational inheritance of addictive-like behaviors. Neuroscience 2014; 264: 198-206

37. Sjoerds Z, Luigjes J, Van Den Brink W, et al. The role of habits and motivation in human drug addiction: a reflection. Front Psychiatry 2014; 5: 8

38. Kennett J, Matthews S, Snoek A. Pleasure and addiction. Front Psychiatry 2013; 4: 117

39. Flanagan O. The shame of addiction. Front Psychiatry 2013; 4: 120

40. Strang J, Babor T, Caulkins J, et al. Drug policy and the public good: evidence for effective interventions. Lancet 2012; 379: 71-83

Correspondence and offprints: Régis Bordet, Département de Pharmacologie Médicale, Centre d'Évaluation et d'Informations sur la Pharmacodépendance, Faculté de Médecine, 1 place de Verdun, 59045 Lille cedex.

E-mail: regis.bordet@chru-lille.fr 